\title{
Erosion-cavitation dans les perçages de refoulement des pompes d'injection de diesels industriels
}

\author{
Erosion-Cavitation in drilling at the industrial diesel injection \\ pumps backflow
}

par D. Jeannerod

Service Technique Moteurs, S.E.M.T. Pielstick Saint-Nazaire

E. Nicole

Service Projets Thermodynamiques, S.E.M.T. Pielstick Saint-Denis

This paper treats one of the difficulties of injection system for Diesel engines: the cavitation. It namely describes how SEMT Pielstick faces erosion-cavitation on running engines, how the source of erosion is understood and the way to avoid it. The lack of knowledge on cavitation fluid and on the kind of cavitation involved an important experimental stage to show the erosion mechanism. After this first stage, a two dimensional calculation of the jet subjected to cavitation including the vapor bubbles was performed. The goal was to be able to forecast the areas which were eroded with a permanent flow in a defined geometry. Finally some solutions to reduce the effect of the cavitation are described. The study of this problem exhibited great difficulties in measurement and an important need of predictive calculation on cavitation.

\section{I 口 INTRODUCTION}

La cavitation est un problème majeur pour les fabricants de systèmes d'injection des moteurs Diesel. Elle se manifeste en de nombreux points du système: pompes d'injection, tuyaux haute pression, porte-injecteurs et pulvérisateurs (fig. 1) [1, 2]. Elle occasionne en particulier des érosions engendrant une réduction de la résistance mécanique de pièces soumises à de fortes contraintes. En pratique on parvient à limiter les effets de la cavitation dans la partie haute pression mais l'érosion se reporte alors avec plus de violence sur les trous de remplissage/décharge.

Le principe de fonctionnement d'une grande majorité de pompes d'injection Diesel est le suivant (fig. 2). Pour assurer une bonne pulvérisation du combustible dans la chambre de combustion, celui-ci doit être injecté à une pression élevée par la pompe d'injection ( $>1000$ bar). Le piston de la pompe a un mouvement alternatif linéaire. En début de profil de came, il referme les orifices de remplissage/décharge et commence à comprimer le combustible dans la chambre haute pression. La pression augmentant, elle parvient à la valeur de tarage du clapet de refoulement qui s'ouvre. Une onde de pression se propage alors à la vitesse du son $(\sim 1400 \mathrm{~m} / \mathrm{s})$ vers l'injecteur. Dès que le niveau de pression atteint la valeur de la pression de tarage de l'injecteur (320 bar), l'aiguille se soulève et libère la sortie du combustible vers les trous du pulvérisateur et la chambre de combustion. La phase d'injection proprement dite commence alors, le débit engendré par la vitesse et la section du piston passe à travers les petits trous de l'injecteur, la pression monte et atteint la pression d'injection ( $>1000 \mathrm{bar}$ ). Le piston qui continue à monter, découvre ensuite par l'intermédiaire de sa rampe inférieure les orifices de remplissage/décharge. A ce moment, la pression régnant dans le circuit haute pression chute rapidement ce qui referme le clapet de refoulement. La pression dans le tuyau diminue jusqu'à la fermeture de l'injecteur. La durée d'injection varie pour nos moteurs de $6 \mathrm{~ms}$ à $14 \mathrm{~ms}$ et elle se répète à une fréquence allant de 4 à 10 Hertz.

Ensuite le piston atteint en décélérant le Point Mort Haut de sa course puis redescend. Les orifices de remplissage/ décharge jusqu'alors ouverts sont fermés à nouveau. Pendant la descente du piston la pression dans la chemise diminue jusqu'à atteindre la pression de vapeur saturante du gazole. Une partie de celui-ci se vaporise. Nous appellerons phase de remplissage le moment où le piston en descendant découvre les orifices de remplissage/décharge par l'intermédiaire du dessus de sa tête. Le gazole extérieur à la chemise entre alors pour y remplacer la vapeur. La pression autour de la chemise pendant cette phase est d'environ 3 bar. Cette pression subit des fluctuations générées par les pompes voi- 


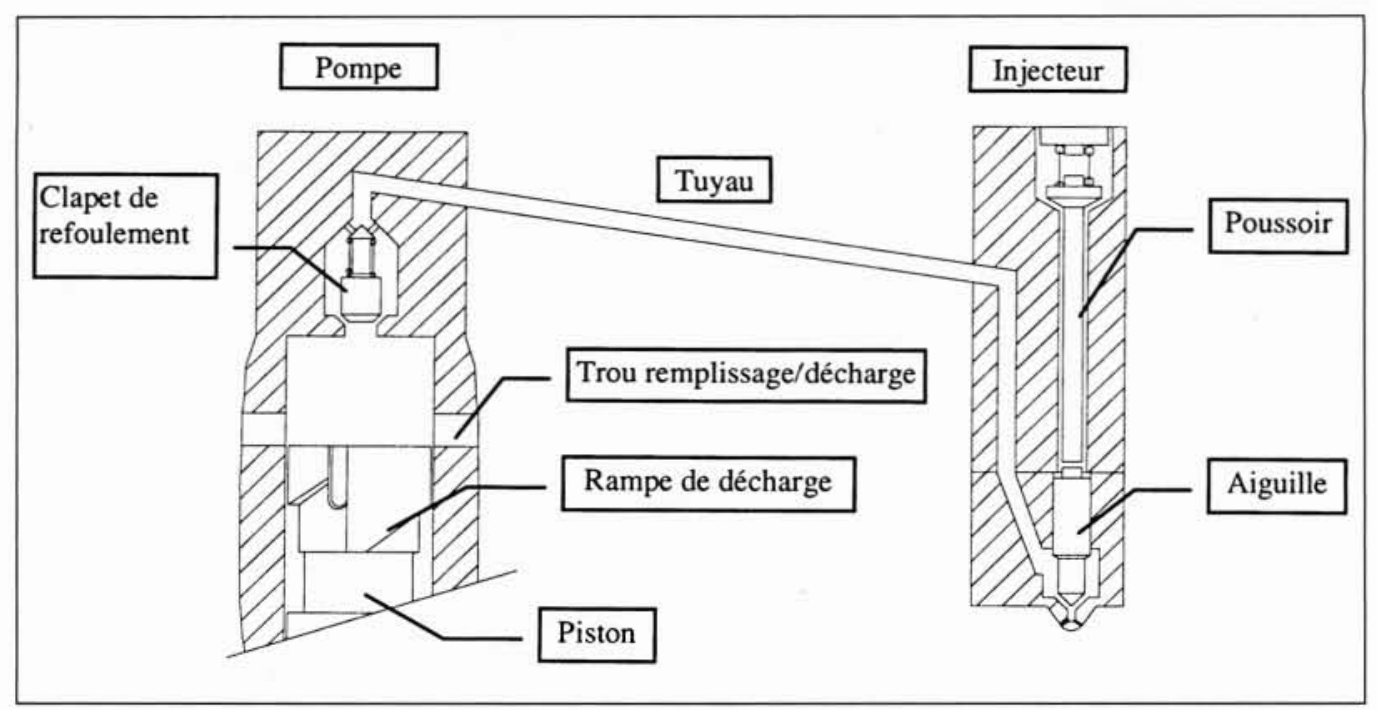

1. Circuit d'injection.

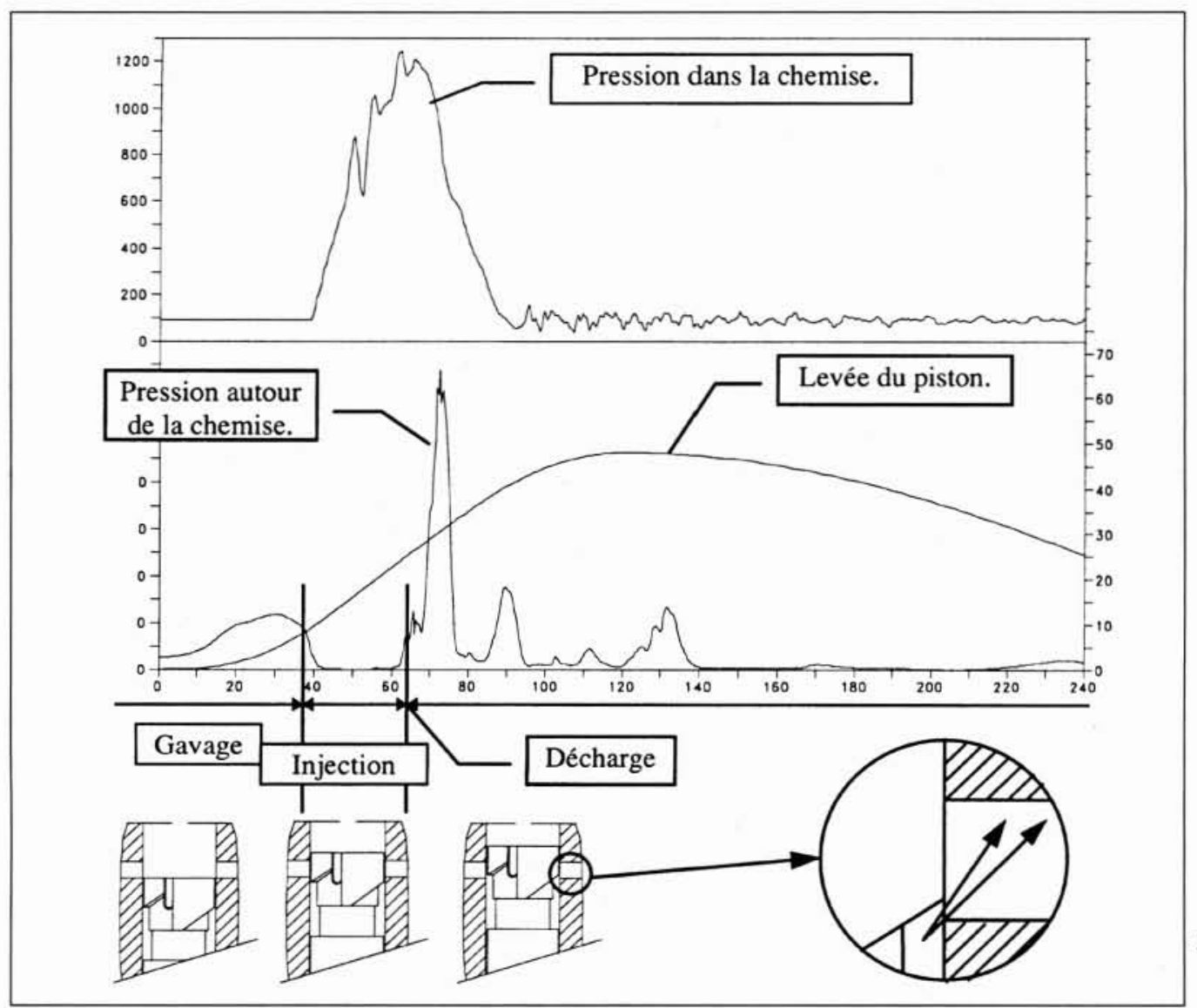

2. Fonctionnement de la pompe d'injection.

sines. Le phénomène de recompression de la vapeur qui suit l'entrée du gazole dans la chemise est mal connu. C'est une des phases critiques où l'on a à la fois présence de vapeur et de liquide.

Nous appellerons phase de décharge le moment où les orifices de remplissage/décharge sont ouverts et engendrent la chute de pression de fin d'injection. A ce moment, typiquement la pression dans la chemise est de l'ordre de $1200 \mathrm{bar}$, ce qui engendre une vitesse à la décharge de $530 \mathrm{~m} / \mathrm{s}$. La pression à l'extérieur de la chemise est de l'ordre de 3 bar en valeur moyenne au moment de la décharge. On constate que le jet se mélange à un fluide sans vitesse, on fait apparaitre ainsi de la cavitation de mélange
[3]. On peut définir le paramètre de cavitation $\sigma=\frac{P_{\text {aval }}-P_{\text {vapeur }}}{\frac{1}{2} \rho V^{2}}$, caractéristique de l'apparition de la cavi-

tation. La valeur limite d'apparition est difficilement estimable car elle dépend, comme la valeur de la tension de vapeur, du mode expérimental employé $[4,5]$. Pour un jet de $6,35 \mathrm{~mm}$ de diamètre, on trouve respectivement un $\sigma_{\text {limite }}$ de 0,27 chez Ooi et de 0,55 chez Lienhard. Dans notre cas, pour $P_{\text {vapur }}=0,06 \mathrm{bar}$, on obtient $\sigma=2,46 \cdot 10^{-3}$. Notre $\sigma$ est très inférieur aux valeurs précédentes donc nous sommes largement dans la cavitation. 


\section{II — INCIDENTS RENCONTRÉS}

En fonctionnement, il s'est avéré que dans certaines conditions les pompes pouvaient subir de fortes érosions à l'intérieur des trous de décharge. Après une durée de fonctionnement de 6000 heures environ, ces érosions peuvent conduire à la fissuration de la chemise et rendent la pompe inutilisable. Les érosions constatées sur les trous ressemblent à celles que subissent habituellement les vis brisejet qui se trouvent en face des trous de décharge. Les faciès d'érosion dépendent fortement du matériau et du traitement qui lui est appliqué. Dans le cas d'un traitement de durcissement superficiel, le faciès ressemble en fait à de petites cavités. Cela se comprend bien, car après avoir percé la couche traitée (cémentation, nitruration) l'érosion attaque sans peine le matériau traité à cour $(800 \mathrm{MPa}$ au lieu de $2000 \mathrm{MPa}$ équivalent pour la couche superficielle) (fig. 3). Le temps de formation de ces cavités dépend du type de traitement. Le changement de matériau ou de traitement peut retarder l'érosion et donc l'échéance de la rupture. La localisation de l'érosion reste identique. Elle se situe près de l'alésage principal de la chemise dans l'orifice de remplissage/décharge.

Le problème de l'érosion de la chemise réside dans le fait qu'elle génère une concentration de contrainte importante à un endroit déjà mécaniquement fortement sollicité. L'érosion crée une rupture de type fragile de l'épaisseur traitée de la chemise. A partir de cette rupture, une fissure peut se développer jusqu'au haut de la chemise. Cette rupture due à une fatigue progressive de la chemise (fig. 4) est de type ductile puisqu'elle se situe au cœur du matériau dont la résistance mécanique est faible comparée à celle de la couche superficielle. L'érosion et la rupture par fatigue sont deux problèmes différents. Chaque ligne frontale représentant un cycle de pompe, on peut évaluer la vitesse de propagation de la fissure. Une analyse microscopique est nécessaire pour voir l'ensemble des lignes frontales. On peut voir la vitesse de propagation de la fissure avec le temps en remarquant le changement de couleur dû au temps d'exposition au gazole.

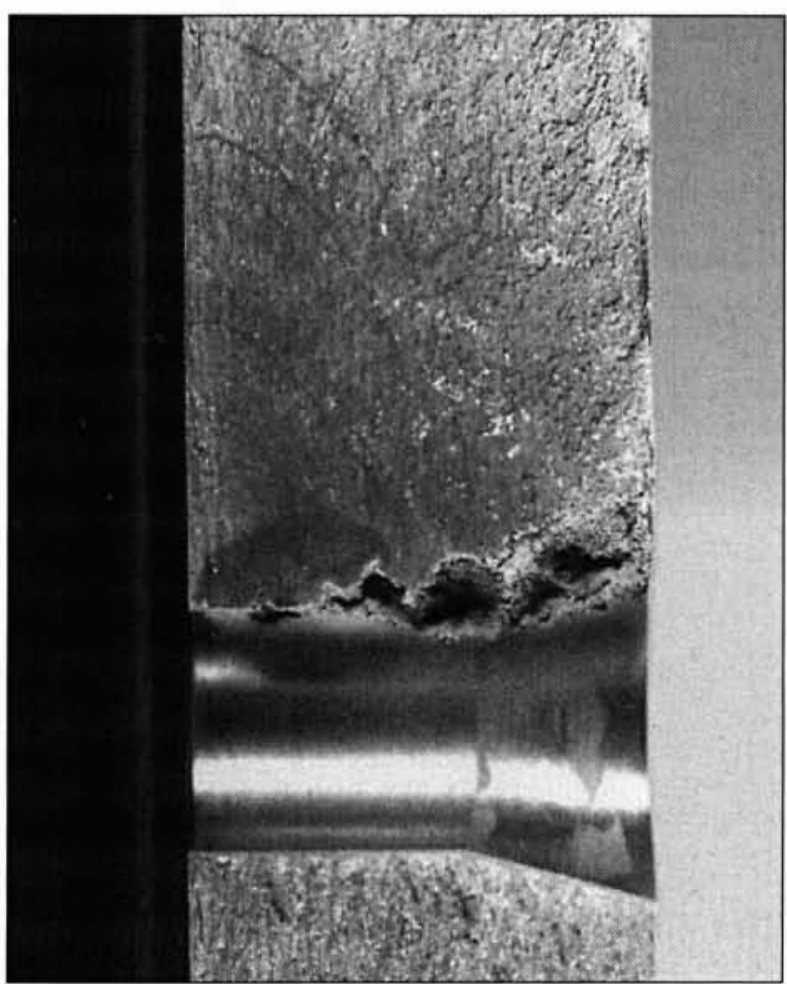

3. Erosion de cavitation dans l'orifice de remplissage/ décharge.

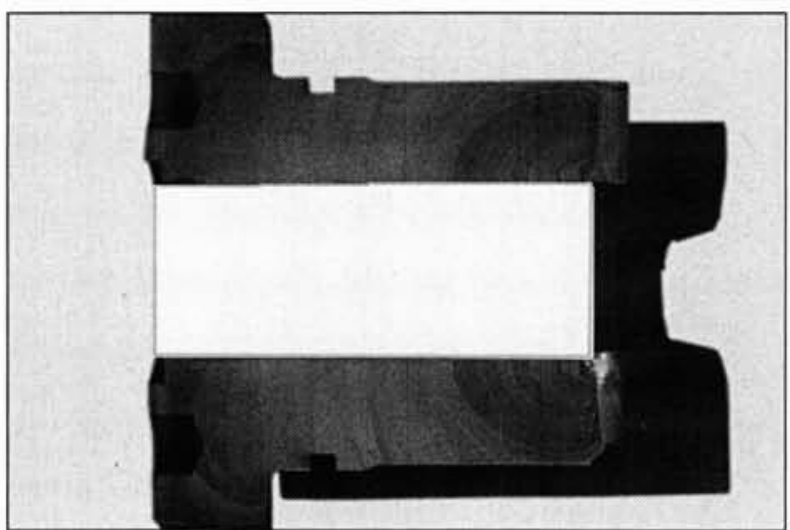

4. Lignes frontales de la fissure.

On ne lutte pas directement contre la rupture en fatigue de la pièce mais contre l'érosion initiale responsable de la rupture de la couche traitée. La conséquence néfaste de cette fissure est que la fuite ainsi créée empêche progressivement la mise en pression de la pompe et provoque donc la coupure de l'injection du cylindre concerné.

On constate également que l'importance de l'érosion dépend fortement du type de combustible. En effet, pour un même nombre d'heures et les mêmes conditions de fonctionnement, les moteurs brûlant du Fioul Lourd (viscosité supérieure à $180 \mathrm{cSt}$ à $50^{\circ} \mathrm{C}$ ) subissent beaucoup moins d'érosions que ceux brûlant du gazole (viscosité inférieure à $9,5 \mathrm{cSt}$ à $50^{\circ} \mathrm{C}$ ). Il faut toutefois garder à l'esprit que sur moteur, on augmente la température du combustible pour ajuster sa viscosité, ce qui diminue la différence entre les types de combustible de ce point de vue tout au moins. Une caractéristique importante dans la cavitation est la pression de vapeur saturante du fluide. Cependant celle-ci dépend du mode expérimental utilisé pour sa détermination. Les conditions dynamiques, les retards thermiques et d'autres paramètres de l'écoulement peuvent modifier les caractéristiques du fluide [6]. La cavitation peut apparaître pour des valeurs différentes de la pression de la vapeur saturante usuelle. De plus dans le cas du gazole, la valeur normale est difficile à obtenir, le gazole étant composé de nombreuses espèces chimiques ayant chacune leurs propriétés. A $40^{\circ} \mathrm{C}$ la pression de vapeur du gazole peut varier entre 50 mbar et 120 mbar suivant son origine. Pour cette raison, certaines installations sont plus sensibles au problème d'érosion que d'autres.

\section{III — MÉTHODES D'INVESTIGATION}

Pour définir l'origine de cette érosion de cavitation, différents essais ont été réalisés. Ils avaient pour objet de préciser dans quelle phase du cycle de fonctionnement de la pompe l'érosion avait lieu et à quoi elle était due. En effet les premiers essais avaient mis en évidence que le gazole se vaporisait plusieurs fois par cycle. On ne savait pas quelle était la phase où se produisait l'érosion. D'autres essais avaient pour objet de qualifier la prédisposition des fiouls à la cavitation. Enfin pour tenter de comprendre le phénomène, des calculs de simulation ont été effectués.

\section{- 3.1 Cavitation accélérée}

La première des manipulations consiste à reproduire, sur un banc d'essai d'injection, le phénomène de cavitation. Pour le mettre en évidence, la chemise de la pompe d'injection est munie de douilles particulières insérées dans les trous de remplissage/décharge (fig. 5). Ces douilles en bronze ou en 


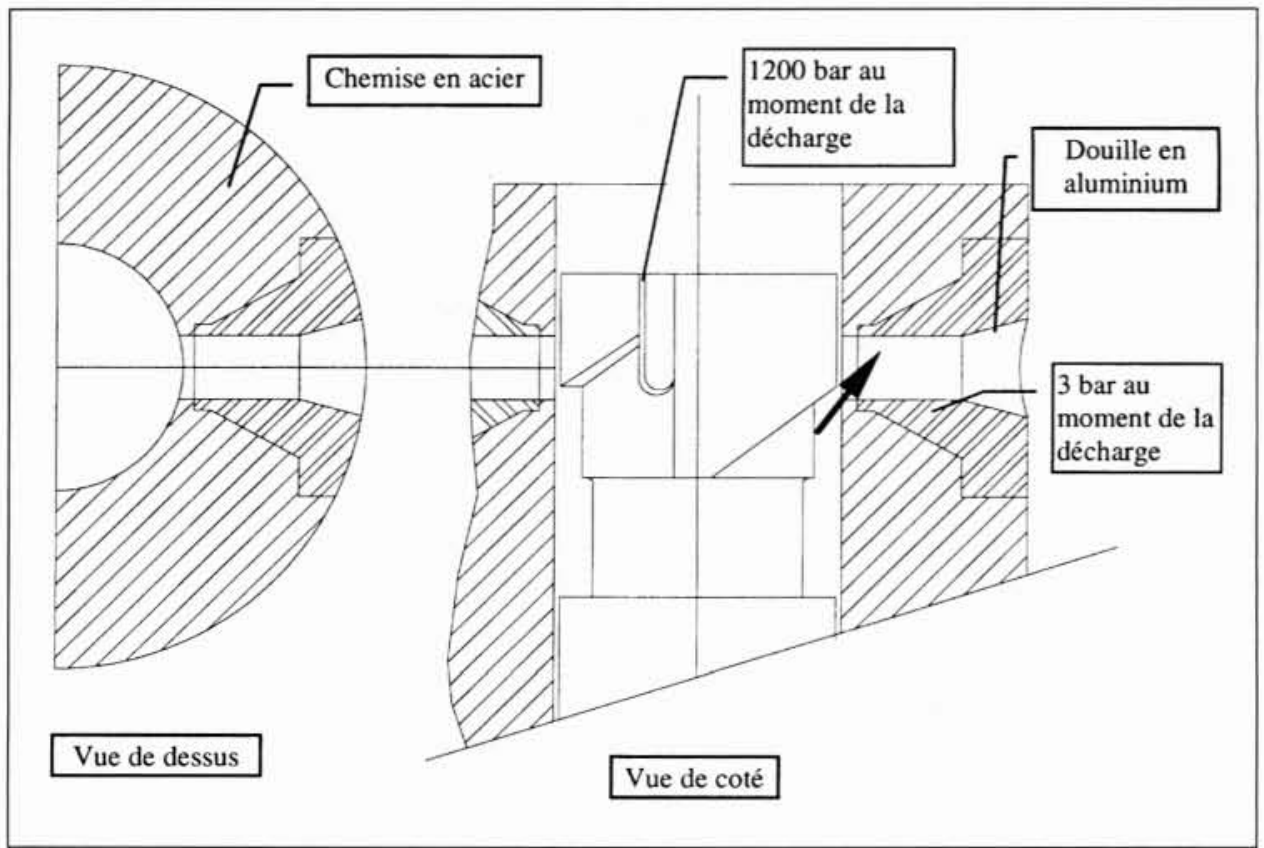
5. Implantation des douilles en aluminium dans la chemise.

aluminium doivent permettre de reproduire en peu de temps l'érosion constatée en service sur les ensembles standards. Le fluide utilisé est du gazole qui est remplacé à intervalles réguliers (voir plus loin). La pompe est munie de nombreux capteurs de pressions afin de vérifier que le fonctionnement au banc est représentatif des conditions normales de fonctionnement moteur. Après une durée de 8 heures, on extrait les douilles pour en faire l'analyse. La différence de masse des douilles avant et après l'essai permet de quantifier l'importance de l'érosion et donc de prédire par comparaison la durée de vie des pièces en fonctionnement. Le profil et la profondeur de l'érosion sont intéressants également pour voir la localisation de l'érosion (fig. 6) et ses implications mécaniques. Les faciès relevés sur ces douilles ressemblant à ceux observés sur les pièces standards, on a considéré l'essai comme représentatif.

Dans un premier temps, on a cherché à identifier la phase de fonctionnement qui occasionne l'érosion. Les deux phases à risques sont : la décharge et le remplissage succédant à la décharge. Pour cela, on commence par faire fonctionner la pompe avec un injecteur plus perméable occasionnant une pression d'injection plus faible que la normale. On constate que l'érosion est beaucoup plus faible donc que la phase remplissage n'est a priori pas responsable des fissurations de chemise. On utilise ensuite un injecteur

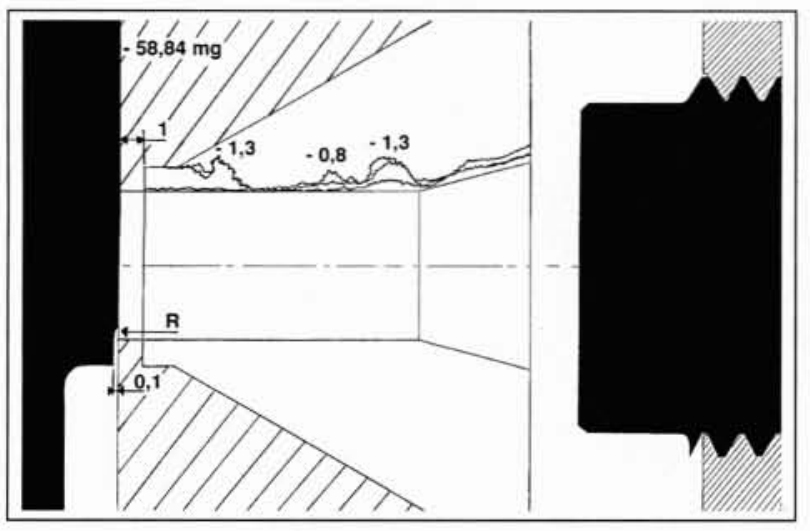

6. Profil d'érosion sur les douilles en aluminium. standard, on constate une forte érosion. On en déduit que la valeur de la pression d'injection est un facteur prépondérant de l'érosion. Après l'identification de la phase dangereuse (phase de décharge), on cherche les différents paramètres agissant sur la perte de masse. Ce sont la géométrie (piston, chemise), la nature du fluide (différents fiouls avec une durée de fonctionnement variable), les conditions de fonctionnement (pression maximum d'injection, pression de gavage). L'âge du fioul semble être un critère important ; en effet, le banc d'essai tournant en boucle fermée, le fioul subit une forme de dégazage. On a ainsi constaté que la cavitation s'amenuisait avec le nombre d'heures de passage du fioul. On peut parvenir à faire disparaître la cavitation avec un gazole "âgé » de 40 heures. L'interprétation que l'on peut faire de ce résultat est que les parties les plus volatiles du gazole se sont évaporées. Cependant lorsque l'on mesure les variations de masse volumique du fluide, on ne constate pas d'augmentation en relation avec la diminution de l'érosion. Cet essai est intéressant parce qu'il est rapide et représentatif mais il nécessite de nombreux ajustements expérimentaux dus au fait que l'on comprend mal la cavitation. Il permet d'essayer de nouvelles géométries et d'estimer les améliorations induites.

\subsection{Prédisposition à la cavitation des fiouls}

Le deuxième essai ayant pour but de caractériser les fiouls vis-à-vis de la cavitation, fait appel à une installation spécifique. Le fioul est placé dans un tube sous pression et s'écoule à travers un diaphragme à la sortie duquel pour une certaine différence de pression, il cavite. A l'aide d'un microphone on enregistre les vibrations acoustiques dues à l'apparition de bulles à la sortie du diaphragme. Deux capteurs de pression donnent la variation de pression entre l'amont et l'aval du diaphragme. Cet essai donne donc une valeur de «limite de cavitation" $\underline{P_{\text {amont }}-P_{\text {aval }}}$ liée à cet

$$
P_{\text {aval }}
$$

essai. La lecture de l'enregistrement du son obtenu lors de l'essai a montré qu'il y avait plusieurs modes d'apparition de bulles. L'amplitude et la fréquence du signal sonore étant différentes pour les deux modes décelés, il laisse penser que deux sortes de cavitation peuvent apparaître dans une telle 


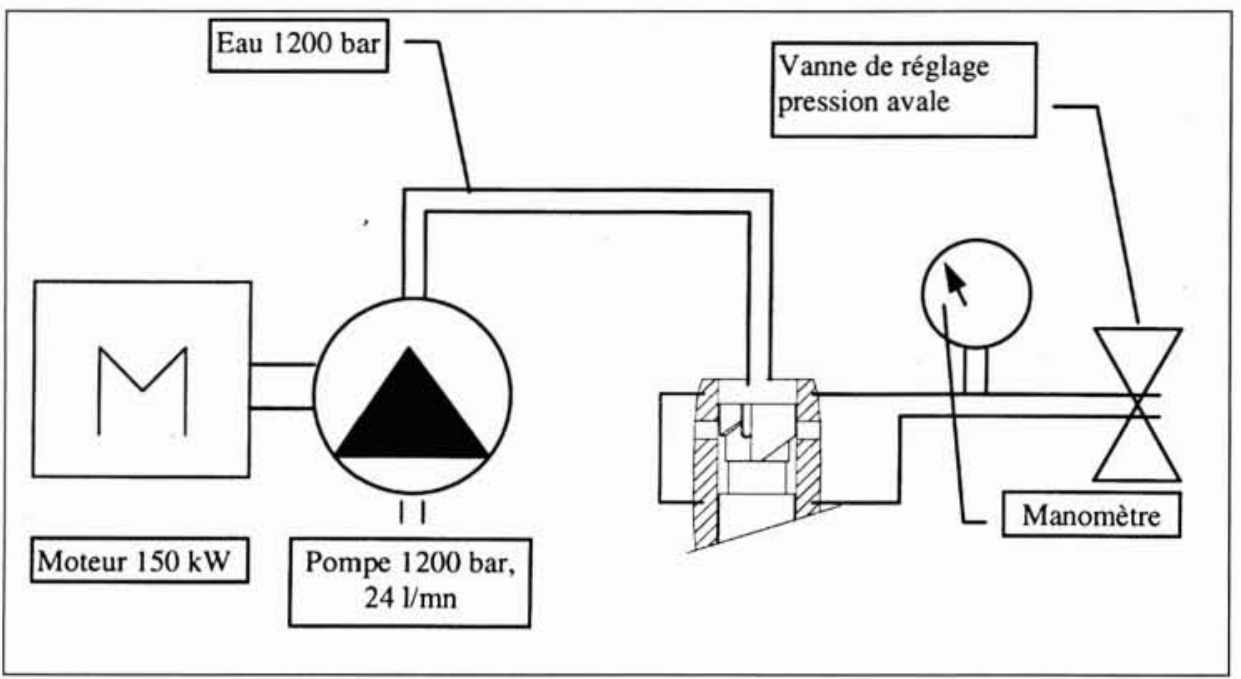

7. Schéma de l'installation de l'essai stationnaire.

installation. Cet essai permet de faire un diagramme de limite de cavitation en fonction de la température du gazole. Les conditions d'écoulement dans l'orifice de remplissage/décharge étant extrêmes en termes de vitesse et de pression, cette caractérisation des fiouls ne permet pas de dire quel est le gazole qui va caviter et celui qui ne le fera pas. Elle permet tout au plus de classer les fiouls a priori par ordre de risque de cavitation.

\subsection{Ecoulement permanent}

Les deux essais précédents ne permettent malheureusement pas de caractériser le moment qui est le plus dangereux dans la phase de décharge. Ils ne permettent pas non plus de tester de nouveaux matériaux ou traitements. Pour y parvenir nous réalisons un écoulement continu simulant la décharge avec du matériel standard. Nous avons découpé en 5 étapes la phase de décharge. Pour chacune de ces étapes nous avons fixé les données géométriques correspondantes et créé un écoulement stationnaire équivalent en termes de vitesse et de pression. En fonctionnement, une phase de décharge ne dure que quelques millisecondes, ce qui permet en écoulement stationnaire de tester un ensemble chemise/piston pour un temps équivalent de plusieurs milliers d'heures de fonctionnement. Le fluide utilisé n'est pas du gazole pour des raisons technologiques mais de l'eau. Le banc est constitué d'une pompe haute pression $(>1000$ bar) (fig. 7), permettant un débit suffisant pour retrouver les conditions de la décharge (de 10 à $30 \mathrm{l} / \mathrm{mn}$ ). Une pompe d'injection standard (donc avec une chemise en acier) dont on peut positionner et bloquer le piston est installée sur un banc d'essais spécifique. On règle la pression autour de la chemise à l'aide d'un robinet pour avoir des conditions proches de celles du moteur. Après quelques heures de fonctionnement, on inspecte les trous de la chemise, le piston et les vis brise-jets (fig. 8). Cet essai permet d'identifier la phase la plus nocive lors de la décharge et de tester de nouveaux matériaux. Les résultats sont toutefois à prendre avec prudence car plusieurs paramètres ne sont pas conformes au fonctionnement normal de la pompe. D'une part le fluide ne correspond pas au gazole $\left(\rho=1000 \mathrm{~kg} / \mathrm{m}^{3}\right.$ au lieu de $850 \mathrm{~kg} / \mathrm{m}^{3}$, viscosité de $1 \mathrm{cSt}$ à $50^{\circ} \mathrm{C}$ au lieu de $3 \mathrm{cSt}$ à $50^{\circ} \mathrm{C}$ ), d'autre part l'écoulement stationnaire ne restitue pas l'énergie de la décharge. Le jet lui-même venant dans notre cas frapper la paroi, il n'est pas à négliger. Cet essai permet d'obtenir rapidement le classement des matériaux et traitements résistant le mieux à la cavitation.

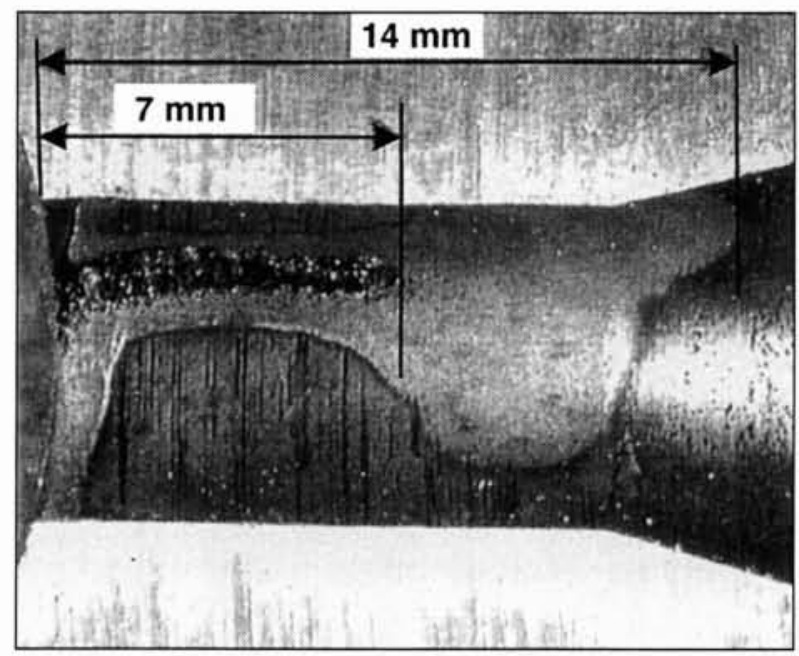

8. Erosion stationnaire sur la chemise.

\section{- 3.4 Modélisation de l'écoulement}

Pour pouvoir prédire l'effet que peut avoir une géométrie sur l'importance de l'érosion de cavitation, des calculs de mécanique des fluides ont été réalisés. Les conditions d'écoulement étant très particulières puisqu'il s'agit principalement d'un jet à grande vitesse $(\sim 500 \mathrm{~m} / \mathrm{s})$, le calcul est effectué par le laboratoire d'usinage non-conventionnel de l'ENSAM qui est spécialisé dans le calcul d'écoulement dans les buses de découpage à l'eau. En effet, le jet de décharge a des caractéristiques proches de celles des jets destinés au découpage à l'eau. Le jet combiné à la cavitation se rapproche des jets chargés d'abrasifs utilisés pour le découpage de matériaux métalliques. Le calcul de l'ENSAM permet d'intégrer dans l'écoulement des bulles dont on suit les trajectoires. Ces bulles n'ont pas d'équation permettant de modéliser leur apparition ou leur disparition. La trajectoire des bulles permet simplement de localiser la zone d'impact préférentielle sur la paroi (fig. 9). Les résultats que nous fournit la simulation sont le taux de présence de bulles sur la paroi en fonction de la distance par rapport à l'alésage principal, ainsi que la vitesse de ces bulles lorsqu'elles atteignent la paroi. $\mathrm{Si}$ l'on considère que les dégâts sont proportionnels à la quantité de bulles « rabattues" sur la paroi avec une vitesse minimum, on peut localiser la zone érodée. Nous ne connaissons pas à l'heure actuelle de modèle d'érosion de cavitation per- 


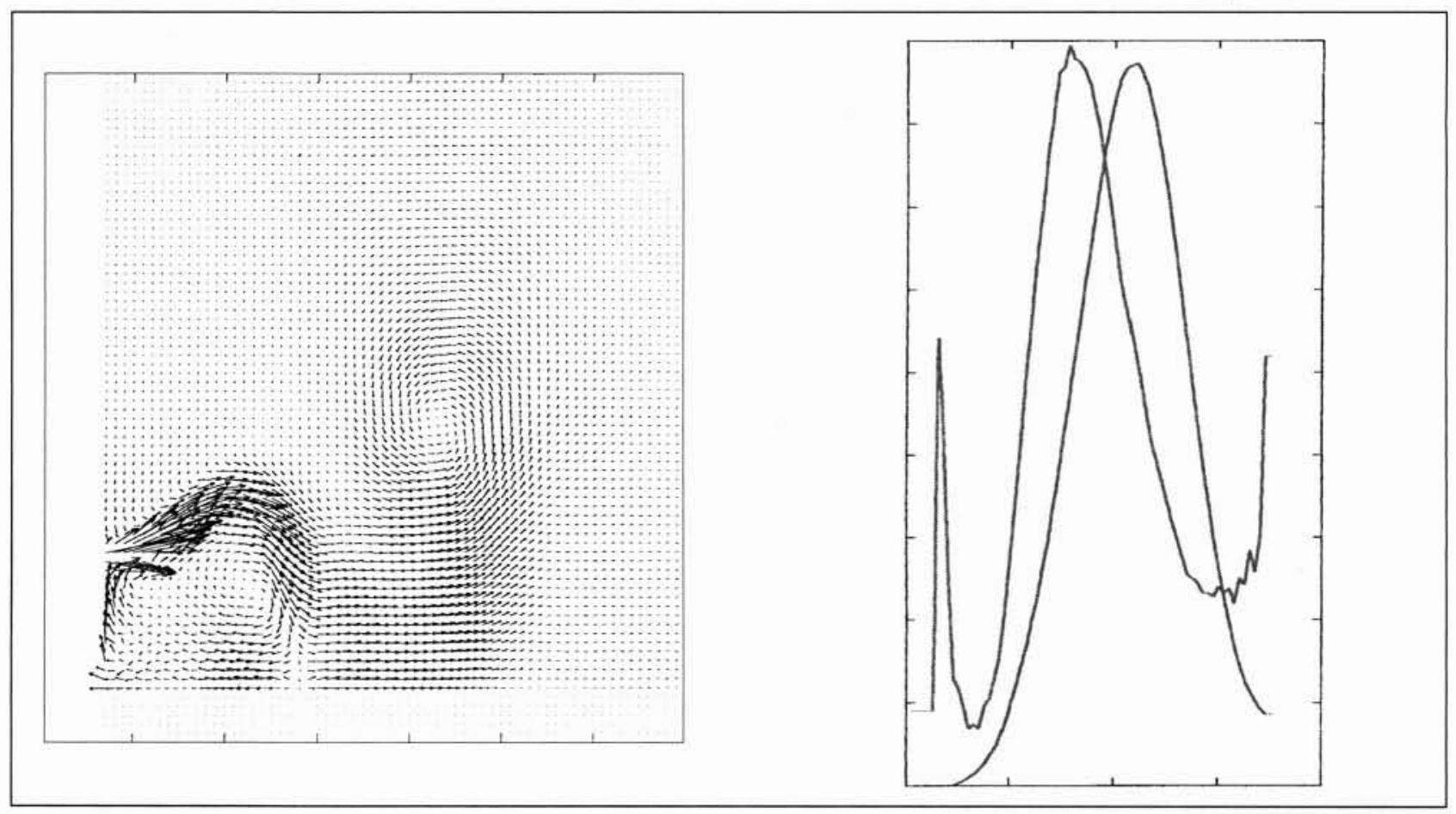

Champ de vitesse dans l'orifice de remplissage/décharge.

Taux de bulles et vitesse sur la paroi.

9. Simulation d'écoulement par l'ENSAM.

mettant de quantifier la perte de matière directement à partir des résultats de la simulation. On ne peut déduire des calculs que la localisation possible de cette érosion en fonction des paramètres géométriques. Un complément envisageable est d'introduire un modèle de développement de la cavitation (par exemple [6]) dans le fluide simulé. Un modèle de la mécanique de l'érosion serait également intéressant pour compléter les simulations d'écoulement.

\section{IV $\square$ SOLUTIONS POSSIBLES}

Les solutions permettant de réduire l'importance de l'érosion de cavitation sont de 3 natures différentes. La première consiste à changer le matériau de la chemise ou le traitement superficiel.
Dans un premier temps seul le traitement superficiel a été changé. Les traitements essayés sont la nitruration et la cémentation. Il est difficile de prévoir le comportement d'un matériau à la cavitation [7] car cela dépend de la manière dont est conduite l'expérimentation. Il est encore plus difficile d'évaluer le comportement d'un matériau dont les caractéristiques sont variables avec la profondeur. Ces chemises sont donc directement essayées sur moteur. Le temps de fonctionnement nécessaire à une érosion significative étant long ( 1 an), nous n'avons pas encore les résultats.

La deuxième solution est basée sur une modification de la géométrie. Pour estimer celles pouvant être intéressantes, nous avons deux possibilités. La première consiste en des essais de cavitation accélérée avec des douilles en aluminium, ce qui nous a permis de définir plusieurs géométries satisfaisantes que nous validons sur moteur actuellement. La deuxième consiste en une définition par le calcul de méca-

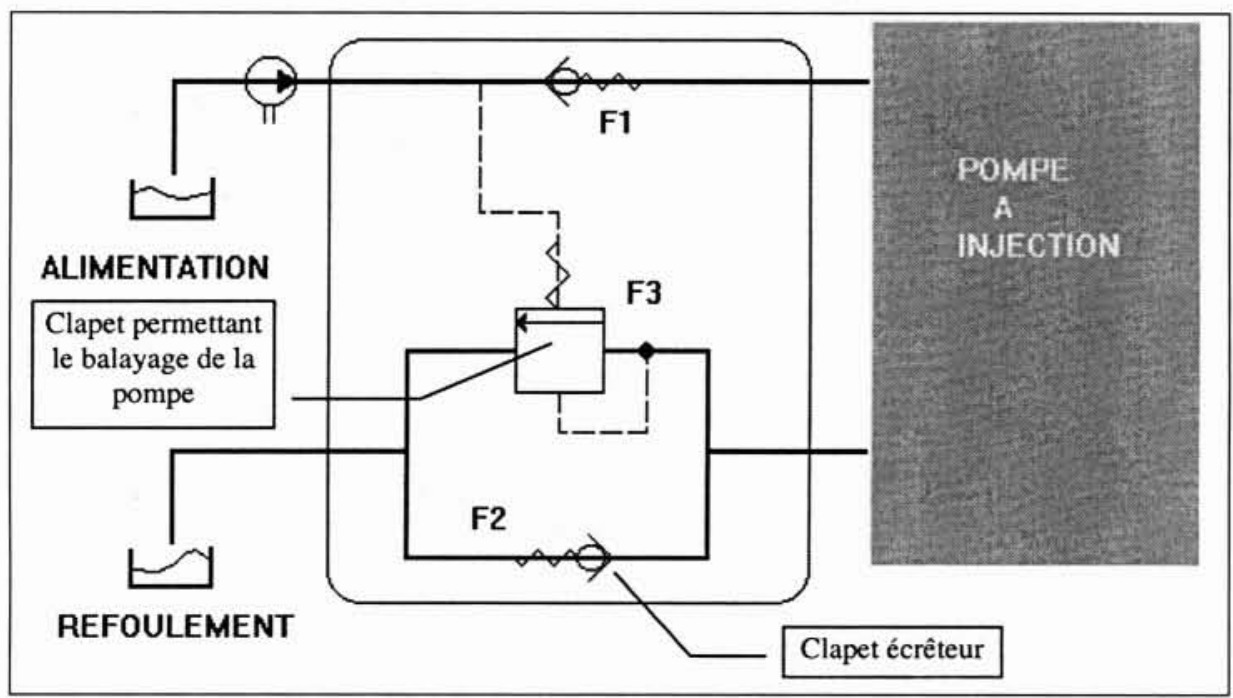

10. Modification des conditions extérieures à la chemise. 


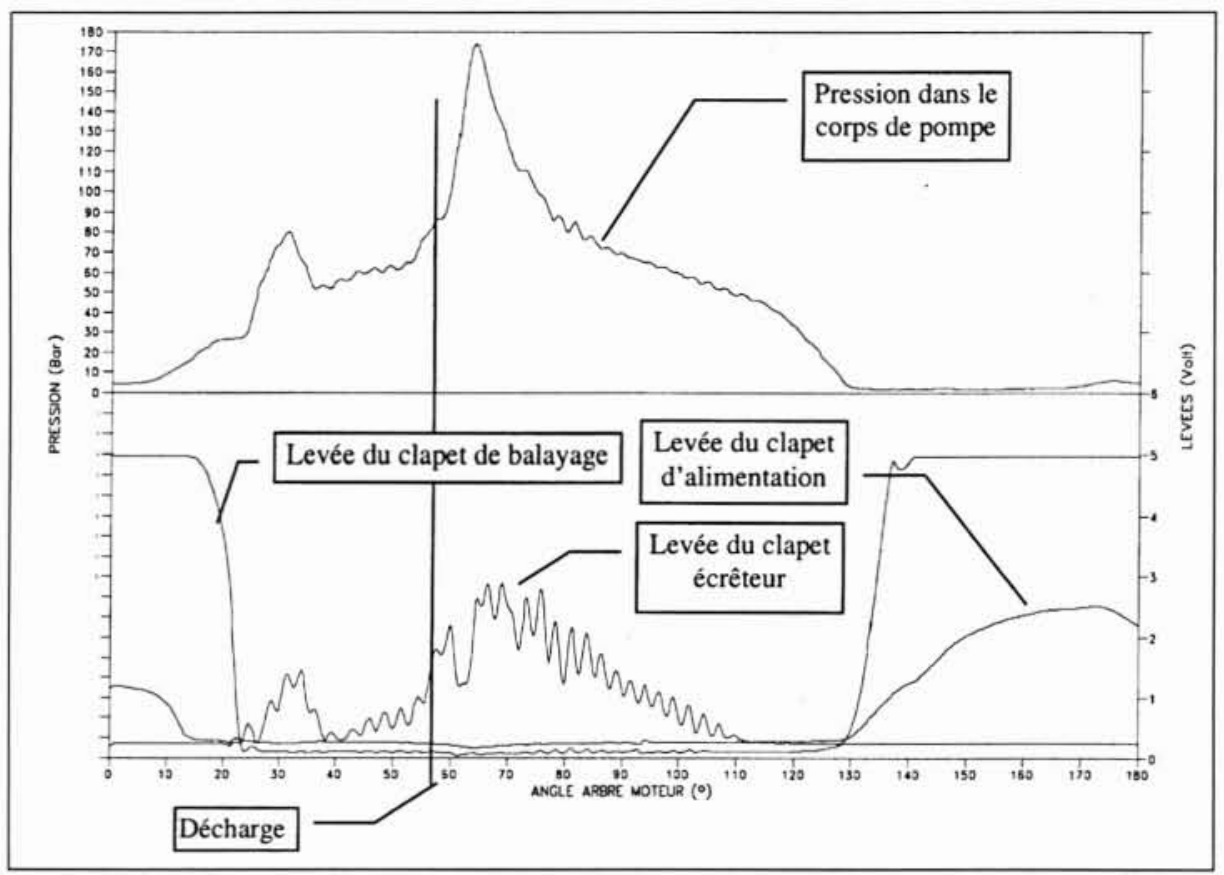

11. Cycle de fonctionnement.

nique des fluides avec transport de bulles de l'ENSAM. Ce calcul est ensuite complété par un essai de cavitation accélérée pour le valider; la prédiction numérique permet de diminuer les tâtonnements mais pas encore de se passer de validations. Si l'essai est concluant, la géométrie ainsi définie est appliquée à un ensemble d'injection et testée sur moteur. A nouveau ces essais prennent beaucoup de temps pour avoir un résultat significatif avec les matériaux normaux.

La troisième possibilité consiste en une modification des conditions aux limites de l'environnement du jet de décharge. En pratique, on tente par l'intermédiaire de clapets de maintenir une pression relativement élevée dans le trou de remplissage/décharge (fig. 10). Le clapet anti-retour à l'alimentation (F1) impose le sens de l'écoulement de l'alimentation au refoulement et participe par sa fermeture à l'isolement et à la mise en pression du corps de pompe. Un clapet écrêteur (F2) est chargé de maintenir la pression au sein du corps de pompe au moment de la décharge à une valeur fonction de son tarage. Le clapet de balayage (F3) permet le renouvellement du fluide dans le corps de pompe. Il est conçu pour s'ouvrir lorsque la pression d'alimentation ajoutée au tarage du ressort est supérieure à la pression dans le corps de pompe (cycle fig. II). L'objectif est d'obtenir une différence de pression plus faible, un jet moins violent et une quantité de bulles produites plus faible. A titre expérimental on a montré que le tarage du clapet écrêteur à une pression de 80 bar dans ce trou fait disparaitre quasiment complètement l'érosion de cavitation obtenue avec les conditions habituelles ( 3 bar). La perte de masse est réduite de $95 \%$. La vitesse du jet est alors $510 \mathrm{~m} / \mathrm{s}$ donc sensiblement constante mais le facteur de cavitation $\sigma$ est de 0,07 soit 30 fois plus que la valeur actuelle sur moteur. Cette expérience valide le principe de montée en pression du corps de pompe avant décharge comme un moyen efficace de lutte contre l'érosion de cavitation.

\section{$\mathrm{V} \square$ CONCLUSION}

Les concepteurs et fabricants de pompe d'injection sont souvent soumis à un important problème de cavitation. Celle-ci limite l'augmentation des pressions d'injection qui autoriserait une réduction de la durée d'injection et une amélioration de la combustion. L'érosion engendrée par la cavitation entraîne dans certains cas la rupture des chemises de pompes d'injection. Pour l'instant les connaissances sur la cavitation de mélange ne permettent pas d'empêcher de façon prédictive cette érosion :

- pas de modèle réaliste du jet de décharge créant les bulles de cavitation ni de l'érosion engendrée,

- connaissances théoriques insuffisantes sur la cavitation des hydrocarbures en régime transitoire,

- moyens d'analyse inadaptés lorsqu'ils sont d'application industrielle ou trop onéreux ou complexes à mettre en cuvre quand ils sont de type laboratoire.

Malgré tout, la méthodologie et les expériences décrites précédemment nous ont permis de définir des ensembles d'injection susceptibles de résister à l'érosion de cavitation de manière raisonnable. Par ailleurs, la cavitation est utilisée en permanence par les utilisateurs de moteurs Diesel. En effet, la transformation du combustible en vapeur est obtenue par cavitation du jet à sa naissance à la sortie du pulvérisateur !

\section{RÉFÉRENCES}

[1] BASTENHOF D. (1981). - - Risques de cavitation dans les systèmes d'injection des moteurs Diesel ", Revue Française de Mécanique, $\mathrm{n}^{\circ} 80$.

[2] HUBER E.W., SCHAFFITZ W. \& MELCHER K. (1971), "Cavitation wear in fioul injection equipement " CIMAC STOCKHOLM.

[3] LeCOFFre Y. (1994), - « LA CAVITATION, Traqueurs de bulles », Edition HERMES, $\S 8,1$, p. 199.

[4] OOI K.K. (1985), - «Scale effects on cavitation inception in submerged water jets : a new look.», J. Fluid Mech., 151, p. $367-390$.

[5] LienharD N.R. and STEPHENSON J.M. (1966). — « Temperature and scale effects upon cavitation and flashing in free and submerged jets », Trans ASME D : J. Basic Engng., 88, p. 525532.

[6] PAuchet J., Retailleau A., Woillez J. (1992). "The predicion of cavitation inception in turbulent water jets ", FED Vol. 135, Cavitation and multiphase flow forum ASME.

[7] FRANC J.P. - « LA CAVITATION, Mécanismes physiques et aspects industriels », Presses Universitaires de Grenoble. 